\title{
Effects of Single Whole-Body Vibration Training Unit in Elderly and Child Patients with Neurological Disorders
}

\author{
Jaroslav Majerník ${ }^{1, *}$, Miriam Dziaková ${ }^{2}$ \\ 1 Department of Medical Informatics, Faculty of Medicine, Trieda SNP 1, 04011 Košice, Slovak Republic \\ ${ }^{2}$ Louis Pasteur University Hospital in Košice, Trieda SNP 1, 04011 Košice, Slovak Republic
}

\begin{abstract}
The positive effects of whole-body vibration techniques have already been discussed in many scientific papers all around the world. In addition, the training units integrated into therapeutic and rehabilitation procedures have been considered as beneficial and powerful methods used to improve either mobility or physical status of human beings. Some of the benefits that were already published include enhanced muscular strength, physical power, bone density or blood circulation. However, there is still a lack of evidence proving the significant effects of whole-body vibration in patients, e.g. those with neurological disorders. Therefore, the main objective of our study was to investigate immediate, although short time effect of single whole-body vibration training unit on quality of patients' gait kinematics. Because of the nature of neurological disorders and their negative impacts on patients and families and society too, we decided to analyse this effect in two high risk groups of patients. The first one consisted of elderly patients and the second one of children, both suffering from certain type of neurological disorder. The analysis was based on comparison of individual changes in gait parameters obtained before and after application of whole-body vibration.
\end{abstract}

Keywords: human motion analysis; kinematics; whole-body vibration; rehabilitation; patients; neurological disorders.

\section{Introduction}

Whole-Body Vibrations (WBV) represent the mechanical stimuli entering the human organism through feet while standing on a vertically or horizontally oscillating platform. Nowadays, the vibration platforms are commercially available and use mostly vibrations of low frequencies and small amplitudes to stimulate skeletal muscles of lower body parts.

WBV are quite often used to improve sport trainings. Using WBV, an improvement in muscle performance, strength and speed can be achieved in relatively short time [1]. WBV can have positive effects also in the rehabilitation processes of patients with various neuromuscular diseases $[2,3]$. According to [4], WBV is the most amazing and the most successful technology for therapy that has been produced and made available to the public in recent years. It is so beneficial that everyone can feel everyday benefit, unless its use is limited by specific health problems [5, 6]. If WBV therapy is done correctly, then it helps gently and easily increase body condition, health, physical welfare and weight loss in a shorter time than in any other standard physical exercises.

The effects of WBV on muscular performance were thought to be induced by increasing the gravitational load leading to neurogenic adaptation and possibly 
hormonal changes. However, critical changes of motor outputs due to WBV are more often associated with nerve factors such as increasing the sensitivity of the major muscle spindles of afferent fibres resulting in homonymous alpha motor neurons. This may result in increased motor unit involvement, increased activation frequency or improved synchronization followed by increased muscle activity and resulting force, also referred to as tonic vibration reflex.

Some studies suggest that a strong release in the homonymous fibres, caused by the local application of vibrations to the muscle or to the tendon results in a reduction in the tension reflex and the H-reflex of this muscle. Vibrationstimulated improvement of motor relaxation along with concurrent decline of reflex is known as "vibration paradox". Several published papers suggesting that WBV activity improves muscle performance and insufficient change in reflex of patella tendon or significant inhibition of calf muscle H-reflex support this phenomenon. The neuromuscular effects of vertical WBV have also been published following vibrations made in the crouched position or in standing on the platform with the knees bent. However, the extent to which WBV will produce neuromuscular effects without voluntary muscle activity is unknown.

Several studies have also shown improvement in bone properties after the WBV. While WBV effectiveness is still being investigated, scientific approaches should take into account several key factors. Except of others, the bone reconstruction patterns vary depending on age and hormonal status. Therefore, WBV should be designed specifically for the group of investigated subjects $[7,8]$. In addition, performing WBV in individuals with a high risk of osteoporosis may induce a secondary physiological effect such as improved balance and mobility. Furthermore, there is little data in the current literature on the physiological effect of WBV to other organs and tissues. Vibrationinduced systemic hormone action can provide a mechanism that improves skeletal muscles [9]. Last but not least, the most appropriate frequencies, durations and amplitudes of vibrations needed for beneficial responses are not known $[10,11]$. Frequently, neither the type of vibration signal (e.g. the sine) is published.

Due to the variety of neurological health problems of our patients, we focused on two risk groups, which are seniors and children, as parts of the population in which more significant effect to confirm the positive or negative effects of WBV can be expected. In total, 19 senior $[12,13]$ and 8 child patients [14] were included in the pilot study.

\subsection{Groups of Patients}

\section{Material and Methods}

The study was performed on patients with various neurological disorders. The inclusion criteria were ambulatory patients that are able to walk independently, had no cardiovascular disease or epilepsy, and had no prior experience in WBV training. All the patients and/or their legal representatives were informed about the WBV training, about the tests to be realized and about possible risks and benefits of the research. Prior to participation, they gave written informed consent approved together with the study design by the University Hospital Ethics Committee.

The first group of patients consisted of nineteen elderly patients (age $M=66.74$ years, $S D=3.65$ years, 6 males and 13 females). The anthropometric measures were taken and registered into the patients' experimental protocols too. As for the aim of the study, the anthropometric characteristics of lower extremities were preferred, including thigh length (right: $M=44.89 \mathrm{~cm}, S D=2.26 \mathrm{~cm}$, left: $M$ $=44.42 \mathrm{~cm}, \mathrm{SD}=2.14 \mathrm{~cm}$ ), calf length (right: $M=$ $41.89 \mathrm{~cm}, \mathrm{SD}=3.80 \mathrm{~cm}$, left: $M=41.58 \mathrm{~cm}, \mathrm{SD}=$ $3.91 \mathrm{~cm}$ ) and foot length (right: $M=25.74 \mathrm{~cm}, S D=$ $2.82 \mathrm{~cm}$, left: $M=25.89 \mathrm{~cm}, \mathrm{SD}=2.55 \mathrm{~cm}$ ).

The second group consisted of eight child patients (age $M=3.63$ years, $S D=1.48$ years, 2 males, 6 females) with hemiparesis or paraparesis of lower extremities. Also here, the anthropometric measures were taken and registered into patients' experimental protocols, including thigh length (right: $M=21.50 \mathrm{~cm}, \mathrm{SD}=5.32 \mathrm{~cm}$, left: $M=21.25$ $\mathrm{cm}, \mathrm{SD}=5.15 \mathrm{~cm}$ ), calf length (right: $M=23.25 \mathrm{~cm}$, $\mathrm{SD}=3.88 \mathrm{~cm}$, left: $\mathrm{M}=22.88 \mathrm{~cm}, \mathrm{SD}=3.67 \mathrm{~cm}$ ) and foot length (right: $M=15.25, S D=0.89 \mathrm{~cm}$, left: $M$ $=15.00 \mathrm{~cm}, \mathrm{SD}=0.76 \mathrm{~cm}$ ).

\subsection{Methodology of Experiment}

All participants attended a familiarization session before the study and before the tests were performed. No other physical treatment or intervention was realized at least 24 hour before WBV training session. The experimental protocol 
was designed to discover potential immediate response of a single WBV training unit to the quality of gait kinematics. Training sessions were supervised by rehabilitation specialists and measurements as well as WBV sessions were conducted in the same thermally neutral room intended for physical training. All subjects did not engage in any therapeutic or rehabilitation procedures before testing.

The training session started with physical examination and short warming-up walk. Then, the patient's gait was captured and analysed before WBV exposure. Participants were asked to walk at their natural waking speed along the $6 \mathrm{~m}$ long path. After reaching the end point of the path, they were asked to turn back $\left(180^{\circ}\right)$, i.e. to change the direction of gait, and to walk back to the starting point. Then, they turned back again and walked to the end point of the path, where the last turn back was realized and the patients finished walking in starting point of the path. In that sense, the subjects passed the length of walking path four times. WBV session followed one minute after this control gait was captured and analysed. Here, each participant stood in static position on the vibration platform (VibroGym inSPORTline) with no shoes and socks and holding on the device handle. Erected posture with slightly bended knees was required during vibration test. The patients were asked to stop the training in the case of any pain responses to vibration. Duration of one WBV training unit was set to $10 \mathrm{~min}$. Sinusoidal vertical vibration frequency was set to $30 \mathrm{~Hz}$ with amplitude of $2 \mathrm{~mm}$. 1 min rest interval followed after this WBV exposure. Then, the patients walked again and the kinematics of their gait was captured and analysed in the same way as it was before WBV session. Finally, the kinematics obtained before and after WBV training unit was compared to evaluate its effects on patients' gait.

\subsection{Gait Analysis and Statistical Evaluation}

Patients' gait assessment was performed using our marker-free motion analysis system MAFRAN [15]. The system is based on optical motion tracking method in video sequence with neither passive or active markers attached to the patients' bodies. MAFRAN tries to minimize disadvantages of currently available marker-free systems, to bring new possibilities of motion analysis and to offer clinicians cheaper, but no worse alternative preserving all advantages of marker-based motion analysis systems. Here, the patient's gait is captured in sagittal plane using common commercial video camera. Then, the raw record is used in the system to automatically reconstruct motion trajectories of human body anatomical landmarks, i.e. the trajectories of all lower extremity joints and adjacent segments. These trajectories are consequently used to calculate other kinematical parameters for detailed description of patient's gait. Here analysed parameters include positions, velocities and accelerations of individual joints, hip flexion/extension, knee flexion/extension, and ankle plantar/dorsal flexion angles, gait cycle length, gait cycle time, gait cycle velocity, cadence (cycles per minute), stance phase and swing phase of the gait cycle.

All acquired kinematical parameters were analysed individually within the patient and then within the groups of here included patients as well. The kinematical characteristics of the patients were evaluated as differences between right and left side. The hypothesis was based on assumption that these differences should be smaller after WBV training comparing values obtained before WBV training. Otherwise, the WBV will probably have no immediate benefits for gait kinematics. Statistical methods included descriptive statistics and Student's paired t-test and were used to ascertain specific and significant differences. The significance level was set to $P<0.05$.

\section{Results}

WBV session was well accepted by all patients included in this continuing study. No one felt any pain or expressed any problems during WBV exposure. A first analysis was performed with anatomical joint angles of lower extremities in sagittal plane. Individually, no of the patients had the same curves of all tree joints comparing before and after WBV values. At least one joint's trajectory was changed either in positive or negative direction. An example of changes in anatomical joint angles in 68 years old female patient with left leg muscular weakening is shown in Figure 1.

Figure 1a shows hip flexion/extension angle before and after single WBV training unit. A paired t-test determined that the mean decrease of differences ( $M=1.85, S D=4,58, N=51$ ) was significantly greater than zero, $\mathrm{t}(50)=2.88$, two-tail $p=0.006$ ( $95 \% C L=1.288)$, providing evidence that 

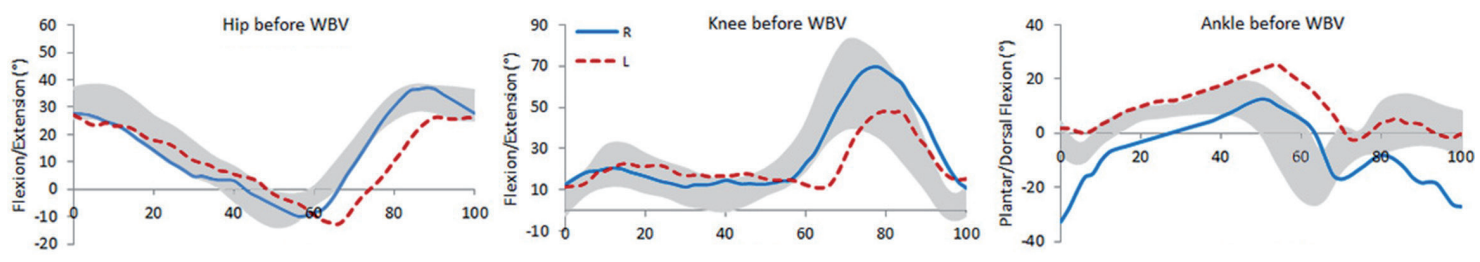

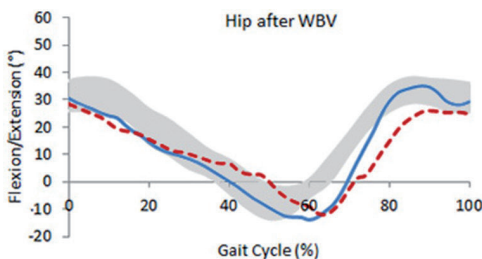

a)



b)

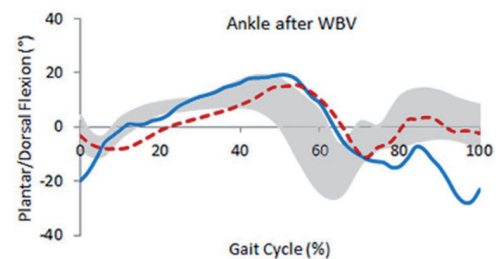

c)

Fig. 1: Anatomical joint angles of 68 years old female patient (a) hip flexion/extension angle before and after WBV exposure, (b) knee flexion/extension angle before and after WBV exposure, (c) ankle plantar/dorsal flexion angle before and after WBV exposure.

the WBV was effective in reduction of differences between right and left hip flexion/extension angle. Figure $1 \mathrm{~b}$ shows knee flexion/extension angle before and after single WBV training unit. Knee flexion/extension angle of the same patient showed that the mean differences between right and left side before and after WBV were decreased, but the mean decrease of differences $(M=-1.61$, $\mathrm{SD}=8,279, \mathrm{~N}=51$ ) was not significantly greater than zero, $t(50)=-1,39$, two-tail $p=0.171$ (95\% $C L=2.329$ ), providing evidence that the WBV was not effective in reduction of differences between right and left knee flexion/extension angle in this patient.

Figure 1c shows ankle plantar/dorsal flexion angle before and after single WBV training unit. The mean decrease of the right and the left side differences in ankle plantar/dorsal flexion angle of the same 68 years old female patient $(M=$ $-12.88, \mathrm{SD}=7,738, \mathrm{~N}=51$ ) was significantly greater than zero, $\mathrm{t}(50)=-11.89$, two-tail $\mathrm{p}=0.000(95 \%$ $\mathrm{CL}=2.176)$ provided evidence that the WBV was effective in reduction of differences between right and left ankle plantar/dorsal flexion angle.

Anatomical joint angles were analysed in all elderly participants of this group in the same way. The summary of right and lefts side differences in anatomical joint angles in elderly patients showed that the most significant changes were registered in the ankle plantar/dorsal flexion angle (89.47\%), followed by hip flexion/extension angle (73.68\%) and knee flexion/extension angle (57.89\%). However, these significant changes include both the positive and the negative changes. The only significant positive changes were chiefly in hip flexion/extension angle (63.16\%) followed by the knee flexion/extension angle (47.37\%) and ankle plantar/dorsal flexion angle (47.37\%). Individually, there were thirteen patients (68.42\%) who had at least two significant positive changes of these kinematical parameters or they had no significant negative changes (subjects 1, 2, 3, 5, 8, 9, 10, 13, $14,15,17,18$ and 19). Two patients (10.53\%) had no beneficial improvements resulting from applied WBV exposure (subjects 6 and 12) and four patients (21.05\%) of the elderly patient subgroup registered worsening because of no significantly positive or only significantly negative changes (subjects 4, 7, 11 and 16).

An example of anatomical joint angles changes in 6 years old female patient with left paraparesis is shown in Figure 2.

Figure $2 \mathrm{a}$ shows hip flexion/extension angle before and after single WBV training unit. A paired t-test determined that the mean decrease of differences $(M=-1.88, S D=5.099, N=51)$ was significantly greater than zero, $\mathrm{t}(50)=-2.63$, two-tail $p=0.000(95 \% C L=1.434)$, providing evidence that the WBV was effective in reduction of differences between right and left hip flexion/extension angle. Figure $2 b$ shows knee flexion/extension angle before and after single WBV training unit. A paired t-test determined that the mean decrease of differences ( $M=2.17, S D=8.213, N=51)$ was not significantly greater than zero, $\mathrm{t}(50)=1.89$, two-tail $p=0.065$ (95\% CL $=2.310$ ), providing evidence 

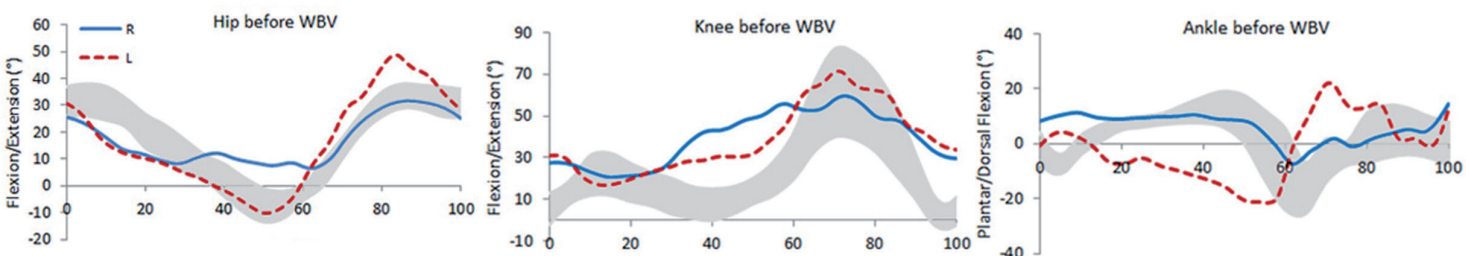

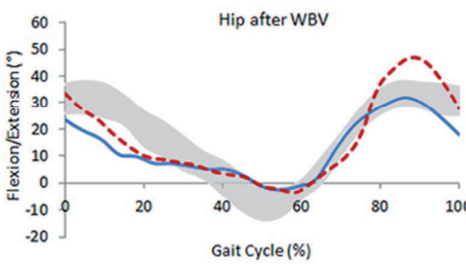

a)



b)

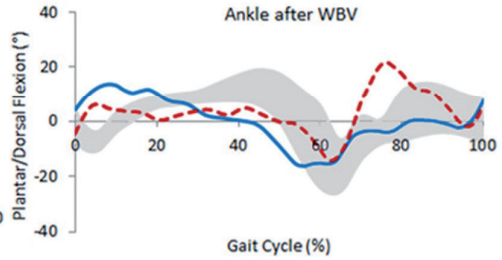

c)

Fig. 2: Anatomical joint angles of 6 years old female patient (a) hip flexion/extension angle before and after WBV exposure, (b) knee flexion/ extension angle before and after WBV exposure, (c) ankle plantar/dorsal flexion angle before and after WBV exposure.

that the WBV was not effective in reduction of differences between right and left knee flexion/ extension angle.

Figure $2 \mathrm{c}$ shows ankle plantar/dorsal flexion angle before and after single WBV training unit. A paired t-test determined that the mean decrease of differences ( $M=3.47, \mathrm{SD}=4.858, \mathrm{~N}=51$ ) was significantly greater than zero, $\mathrm{t}(50)=5.10$, twotail $p=0.000$ ( $95 \% C L=1.366)$, providing evidence that the WBV decreased differences (worsening) between right and left ankle plantar/dorsal flexion angle in this patient.

Anatomical joint angles were analysed in all child patients. The summary of right and lefts side differences in anatomical joint angles in child patients showed that the most significant changes were registered in the ankle plantar/dorsal flexion angle (100.00\%), followed by hip flexion/extension angle (75.00\%) and knee flexion/extension angle (75.00\%). However, these significant changes include both the positive and the negative changes. The only significant positive changes were recognized in ankle plantar/dorsal flexion angle (75.00\%), followed by the knee flexion/extension angle (50.00\%) and hip flexion/extension angle (50.00\%). Individually, there were four patients (50.00\%) who had at least two significant positive changes of these kinematical parameters or they had no significant negative changes (subjects 1, 3, 4 and 6). Two patients (25.00\%) had no beneficial improvements resulting from applied WBV exposure (subjects 2 and 7) and two (25.00\%) of the child patients registered worsening because of no significantly positive or only significantly negative changes (subjects 5 and 8 ).

Figure 3 shows the distribution of statistically significant changes in anatomical joint angles of lower limbs obtained immediately after application of single WBV training unit in groups of senior and child patients enrolled in a pilot study.

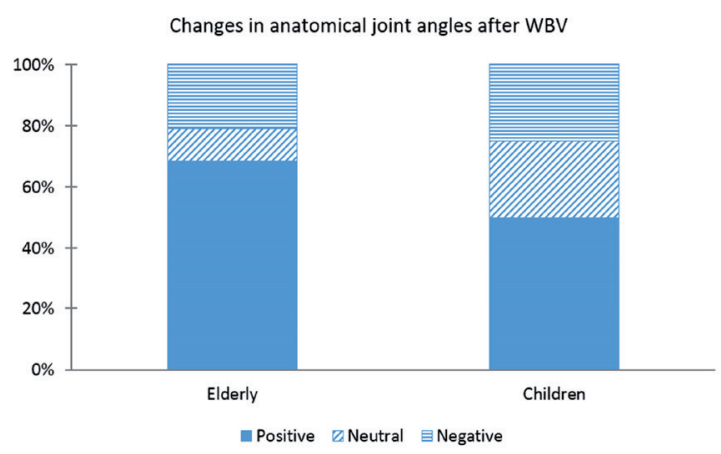

Fig. 3: Percentage of statistically significant changes in anatomical joint angles.

After application of WBV training unit, there were registered mostly positive changes or statistically not significant changes. In both groups of studied patients, the statistically significant negative changes in anatomical joint angles were in 20\% and $25 \%$ respectively. The observed effect of WBV is, as described earlier, in short-term, but these results suggest that individually planned trainings and targeted WBV applications can affect the symmetry of patient movement at the level of 
changes in anatomical joint angles.

\section{Conclusions}

The results of the conducted study confirmed the effect of whole-body vibrations on kinematics of patients' movement. The direct short-term benefit of the WBV training unit was present in more than half of all involved patients. On the other hand, four patients have no statistically significant changes in kinematics considering the condition before and after the application of WBV training unit. Six patients experienced a worsening of one or more analysed parameters after WBV. The short-term effect of WBV was slightly more effective in seniors than in child patients. The results could have been affected by various neurological diseases of here involved patients, such as multiple sclerosis, Parkinson's disease, cerebral palsy or radicular neuritis. Positive sides of WBV are widely discussed and may dependent on many factors. First, they depend heavily on the patient's health, the severity of his/her illness, or the supportive therapeutic procedures. Other factors include muscle activity, status and activities performed during WBV application, optimal vibration frequency and amplitude.

As we expected, the single whole-body vibration training unit had only short-time effect as several patients were analysed one week after the experiment and their kinematics was similar to the pre-exposure status. Utilization of WBV training in patients with neurological disorders may result in benefits for kinematics of human motion, but its significance and mechanism remains unclear and undiscovered. The results of this pilot study provided invaluable data for rehabilitation specialists as well as for development of further research programs in physiotherapy. Based on the results we obtained, it was also confirmed that an individual WBV training and supervised functional treatment should be specified for particular patient. Further research should be realized to clarify WBV specific benefits. The various training approaches including standing in different static positions, sitting on chair with legs on vibrating platform or performing exercises on the platform during therapy sessions should be investigated as well.

\section{Acknowledgments}

Results presented in this work were obtained with the support of the national agency's grant KEGA 017UPJS-4/2016 "Visualization of education in human anatomy using video records of dissections and multimedia teaching materials".

\section{References and Notes}

[1] Cardinale M., Wakeling J.: Whole body vibration exercise: are vibrations good for you?, Br J Sports Med, 2005, 39, pp. 585-589.

[2] Sayenko D.G. et al.: Acute effects of whole body vibration during passive standing on soleus $\mathrm{H}$-reflex in subjects with and without spinal cord injury, Neuroscience Letters, 482, 2010, pp. 66-70

[3] Baumbach S.F., Fasser M., Polzer H., Sieb M., Regauer M., Mutschler W., Schieker M., Blauth M.: Study protocol: The effect of whole body vibration on acute unilateral unstable lateral ankle sprain - a biphasic randomized controlled trial, BMC Musculoskeletal Disorders, 2013, 14:22.

[4] Cooper J.: The amazing wellness benefits of Whole Body Vibration Therapy, Finally - a comprehensive guide to the facts about whole body vibration therapy, Whole Body Vibration System, 2009, 23 s.

[5] El-Khatib A., Guillon F.: Lumbar intradiscal pressure and whole-body vibration - first results, Clinical Biomechanics, 2001, 16, Supplement No.1, pp. S127-S134.

[6] Gloeckl R. et al:: Effects of whole body vibration in patients with chronic obstructive pulmonary disease - A randomized controlled trial, Respiratory Medicine, 2012, 106, pp. $75-83$.

[7] Prisby R. D., Lafage-Proust M. H., Malaval L., Belli A., Vico L.: Effects of whole body vibration on the skeleton and other organ systems in man and animal models: What we know and what we need to know, Ageing Research Reviews, 7, 2008, pp. $319-329$

[8] González-Agüero A. et al.: Effects of whole body vibration training on body composition in adolescents with Down syndrome, Research in Developmental Disabilities, 2013, 34, pp. 1426-1433.

[9] Shibata N., Ishimatsu K., Maeda S.: Gender difference in subjective response to whole-body vibration under standing posture, International Archives of Occupational and Environmental Health, 2012, 85 (2), pp. 171-179.

[10] Maeda S., Mansfield N.J., Shibata N.: Evaluation of subjective responses to wholebody vibration exposure: Effect of frequency content, International Journal of Industrial Ergonomics, 2008, 38, pp. 509-515.

[11] Guzanová A., Ižaríková G., Brezinová J., Živčák J., Draganovská D., Hudák R.: Influence of build orientation, heat treatment, and laser power on the hardness of Ti6Al4V manufac- 
tured using the DMLS process, In: Metals. Vol. 7, no. 8 (2017), p. 1-17, ISSN 2075-4701

[12] Majerník J., Živčák J.: Effect of Whole Body Vibration on Functional Mobility in Elderly Patients, Acta Mechanica Slovaca, Vol. 17 (3), 2013, ISSN 1335-2393, pp. 64 - 69.

[13] Majerník,J., Dziaková,M., Živčák,J.: Utilization of Single Whole Body Vibration Training Unit in Rehabilitation of Elderly Patients with Neurological Disorders, Proceedings of the 2015 Federated Conference on Computer Science and Information Systems, FedCSIS 2015, 2015F304, pp. 275-279.

[14] Majerník J., Dziaková M., Živčák J.: Rehabilitation in children with neurological disorders using whole body vibration. Information and Digital Technologies 2015, 2015, ISBN 978-14673-7185-8, IEEE Catalog Number CFP15CDT-USB, pp. 235 $-238$.

[15] Majerník J.: Reconstruction of Human Motion Trajectories to Support Human Gait Analysis in Free Moving Subjects, In: Pancerz,K. and Zaitseva,E.: Computational Intelligence, Medicine and Biology; Studies in Computational Intelligence, 2015, 600, pp. 57-77, DOl: 10.1007/978-3-319-16844$9+4$.

\section{Biographical notes}

Jaroslav Majerník, doc. Ing. PhD.: (born 1977) Head of Department of Medical Informatics at Pavol JozefŠafárik University, Faculty of Medicine in Košice. He received his Master's degree in the field of Electronics and Telecommunications at Technical University in Kosice, Slovakia in 2000 and Ph.D. degree in Bionics and Biomechanics in 2005. His professional and research interests include biomedical engineering and medical informatics. Assoc. prof. Majernik is author or co-author of nine books and more than 200 research works and projects in the fields of biomechanics and computer science. He is member of ESMAC, MEFANET Coordination Council, MEFANET Journal Editorial Board, Multimedia Technology Editorial Board and several national societies.

Miriam Dziaková, MUDr. PhD.: she is a Head of Department of Physiotherapy, Balneology and Medical Rehabilitation at Louis Pasteur University Hospital in Kosice. Her professional career and achievements in clinical praxis resulted in the position of chief expert of the Ministry of Health of Slovak Republic for Physiotherapy, Balneology and Medical Rehabilitation. She is also a member of several medical societies in Slovak Republic. 


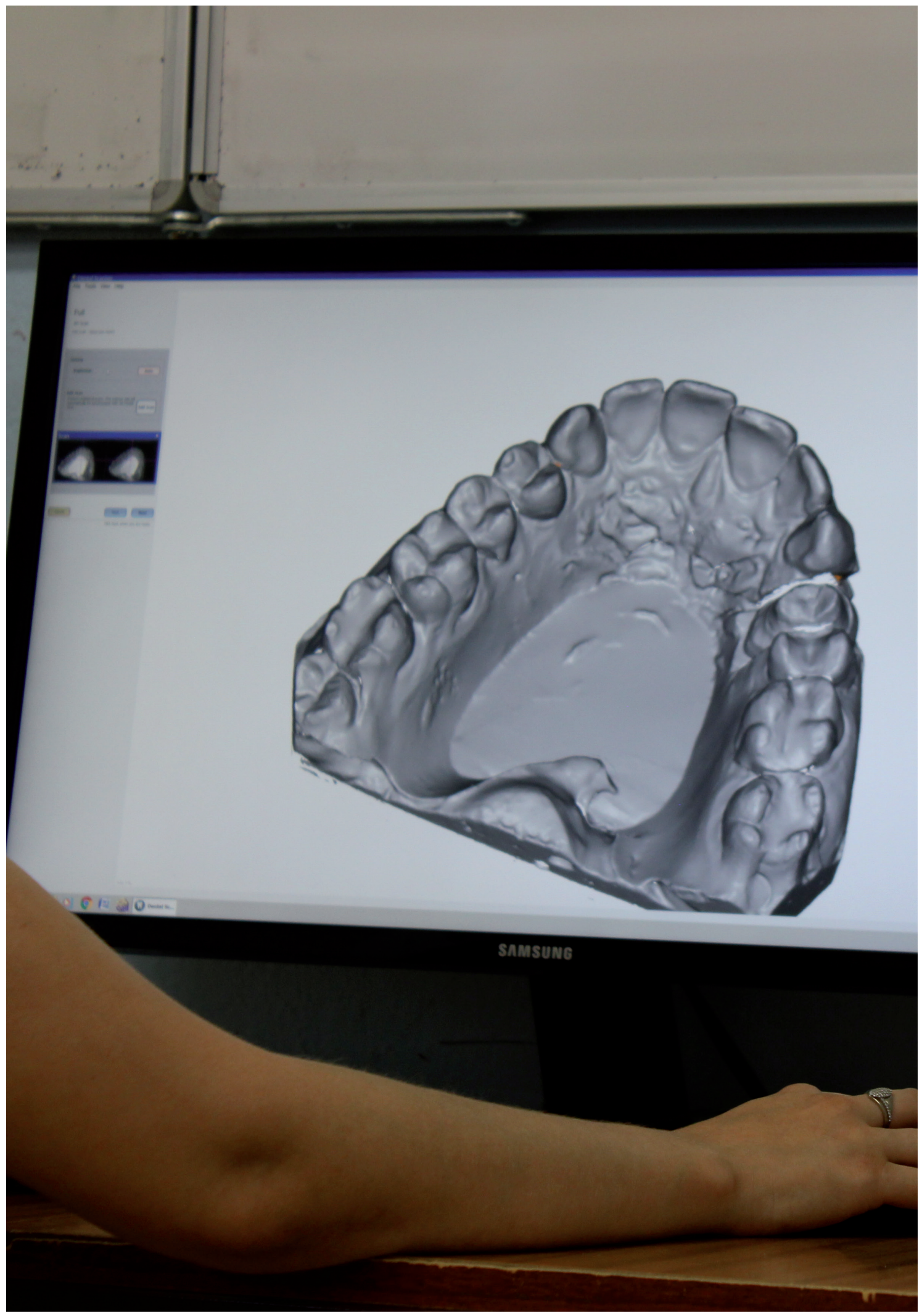

